\title{
Vascular graft infection: Improving diagnosis with functional imaging
}

\author{
Matthieu Pelletier-Galarneau, MD, MSc, ${ }^{\mathrm{a}, \mathrm{b}}$ and Daniel Juneau, $\mathrm{MD}^{\mathrm{c}, \mathrm{d}}$ \\ a Department of Medical Imaging, Institut de Cardiologie de Montréal, Montreal, Canada \\ b Gordon Center for Medical Imaging, Massachusetts General Hospital, Harvard Medical School, \\ Boston, MA \\ c Department of Medical Imaging, Centre Hospitalier de l'Université de Montréal, Montreal, \\ Canada \\ d University of Ottawa Heart Institute, Ottawa, Canada
}

Received Jun 26, 2020; accepted Jun 26, 2020

doi: $10.1007 / \mathrm{s} 12350-020-02269-z$

\section{See related article, https://doi.org/10.10 07/s12350-020-02227-9.}

Positron emission tomography (PET) imaging with fluorodeoxyglucose (FDG) has seen increased applications in the cardiovascular realm over the last decades. Its usefulness is already established in a wide range of conditions, including myocardial viability imaging, assessment of inflammation in diseases such as sarcoidosis and vasculitis, as well as imaging of infectious processes, such as infective endocarditis and cardiac implantable electronic device infection. ${ }^{1-4}$ In addition, FDG-PET is emerging as a useful tool in several other cardiovascular applications such as for the diagnosis of vascular graft infection (VGI). ${ }^{5}$

Vascular prostheses are routinely used to treat vascular aneurysms, dissections, and occlusions. Improvement in graft design and surgical techniques, as well as the advent of less invasive endovascular implantation techniques, have led to an overall increase in the number of procedures and have facilitated their use in patients who are older and have more comorbidities. VGI is a rare but extremely serious complication following vascular graft implantation, with an incidence of approximately $1 \%-5 \% .^{6-9}$ It is associated with considerable morbidity, mortality, and healthcare system costs.

\footnotetext{
Reprint requests: Matthieu Pelletier-Galarneau, MD, MSc, Department of Medical Imaging, Institut de Cardiologie de Montréal, Montreal, Canada; matthieu.pelletier-galarneau@icm-mhi.org

J Nucl Cardiol 2022;29:3450-4.

$1071-3581 / \$ 34.00$

Copyright (C) 2020 American Society of Nuclear Cardiology.
}

Accurate and timely diagnosis is paramount to ensure that appropriate treatment is initiated promptly. Unfortunately, the clinical manifestations of VGI are often non-specific and deceptive. Further complicating the issue is the fact that there are a wide variety of vascular prostheses types and implantation techniques, which can impact the incidence, presentation, and treatment of VGI. Conventional imaging using ultrasound, computed tomography angiography (CTA), and magnetic resonance imaging (MRI) are often used, but all have their limitations. To address these diagnostic challenges, the Management of Aortic Graft Infection (MAGIC) group of experts introduced criteria in 2016 for the diagnosis of aortic graft infection. They comprise minor and major criteria divided in three categories: clinical/surgical, radiology, and laboratory (Table 1). ${ }^{10}$ Notably, some criteria can be considered subjective (e.g., "fever with graft infection as the most likely cause'), while others are non-specific and represent only indirect evidence of vascular graft infection (e.g., abnormally elevated inflammatory markers). Despite these limitations, the MAGIC criteria represent a valuable tool in the evaluation of patients with suspected infection. $^{11}$

The use of FDG-PET in the diagnosis of VGI has been investigated in several small studies and three recent meta-analyses provide a good overview of the state of the literature. ${ }^{12-14}$ The meta-analyses report similar results with excellent pooled sensitivity (93\%, $95 \%$, and $96 \%$ ) and good pooled specificity $(74 \%, 78 \%$, and $80 \%$ ) of FDG-PET for the detection of VGI. In their meta-analysis, Folmer et al also compared FDG-PET and FDG-PET/CT to CTA and white-blood cell (WBC) scintigraphy. They showed that both FDG-PET/CT and WBC scintigraphy outperform CTA, and the authors 
argue that since $\mathrm{WBC}$ is more expensive and timeconsuming, PET might be the best initial imaging technique. ${ }^{14}$ Rojoa et al for their part compared 5 different FDG-PET interpretation methods that can be used for VGI. ${ }^{13}$ They concluded that the 2 best methods were either using the pattern of uptake (focal vs diffuse) or an uptake intensity cut-off $\left(\mathrm{SUV}_{\max }\right)$.

In this issue of the Journal, Zhang et al report the results of a prospective single-center study in which FDG-PET/CT was performed in 35 patients with suspected intra-thoracic aortic VGI. Of the 35 patients included, 25 fulfilled the MAGIC criteria for VGI. They demonstrated a high concordance $(31 / 35)$ between PET findings and the MAGIC criteria for VGI. The reported sensitivity, specificity, and accuracy of FDG-PET/CT interpreted with the visual grading system (VGS) were $96 \%, 70 \%$, and $89 \%$, respectively, using the MAGIC criteria as gold standard. In three cases, FDG-PET/CT was considered positive but the MAGIC criteria for aortic graft infection were not met. In another case, FDG-PET/CT was negative while infection was established by the MAGIC criteria. The results obtained are in agreement with those of other studies using similar methods. $^{15}$

The gold standard for the diagnosis of vascular graft infection is positive cultures from biopsy, aspirated liquid, or surgical tissue. As correctly stated by the authors, these are not always available. In that context, conducting trials evaluating the management and diagnostic options of VGI can be quite challenging. ${ }^{16}$ To address these limitations, the MAGIC criteria are often use as a surrogate gold standard. Based on their results, the authors rightfully claimed that FDG-PET/CT might play a complementary role to the MAGIC criteria. This statement is supported by the very high sensitivity of FDG-PET/CT to detect vascular graft infection. Perhaps just as important, whole-body FDG-PET/CT can detect other foci of infection and inflammation, which could account for non-specific MAGIC minor criteria (e.g., abnormally elevated inflammatory markers). Ultimately, this could result in an improvement of the MAGIC criteria's specificity by providing an alternative explanation for some of the less specific findings that are part of the criteria.

A strength of this study is the use of four different PET parameters: VGS, presence of focal uptake, maximal standardized uptake value $\left(\mathrm{SUV}_{\max }\right)$, and maximal target-to-background ratio $\left(\mathrm{TBR}_{\max }\right)$. The use of a single uptake intensity cut-off such as $\mathrm{SUV}_{\max }$ is insufficient to reached optimal diagnostic accuracy as several biological and technical factors, independent of the disease state, may affect such thresholds. For instance, it has well been established that inflammation at sites of previous surgical interventions is an important confounder when assessing FDG uptake intensity. ${ }^{5,17}$ The VGS is a 5-point visual grading system introduced by Sah et al for the analysis of PET images in the context of VGI (Table 2). ${ }^{18}$ It integrates qualitative reading of uptake intensity and distribution to reach a binary interpretation. Importantly, interpretation of VGS must consider the clinical context, especially in the presence of VGS-3 scans. For example, mild focal uptake may represent sterile post-surgical changes and intense diffuse uptake can be seen several months following surgery. ${ }^{5}$ Interestingly, among the 3 false positive obtained in the current study, 2 were categorized as VGS-3 with diffuse uptake. It has been suggested that multiparametric assessment of FDG-PET images combining both quantitative (SUV/TBR) and qualitative (focal/diffuse) evaluation provides improved accuracy by circumventing some of the limitations of each methods alone. ${ }^{5,15,19}$ Interestingly, in this work, the combination of two parameters provided the highest accuracy. Nonetheless, larger multicentric prospective studies are still required to validate the optimal interpretation parameters.

Another interesting aspect of Zhang et al work is the inclusion of patients who underwent two types of procedure: open chest aortic surgical repair and endovascular aortic repair. They observed inferior specificity of FDG-PET/CT in patients who underwent open surgery (50\%) compared to those with endovascular procedure $(100 \%)$, with all false-positive cases having an open surgery. These findings are not surprising as open chest procedures are significantly more traumatic, which likely leads to more intense and heterogeneous post-intervention FDG uptake. Furthermore, post-intervention uptake may also persist for a longer period following open chest surgery, but this remains to be clearly demonstrated. Finally, open chest procedures often involve the use of biological adhesive, which may lead to persistent focal uptake months after the procedure. This further highlights the importance of evaluating the diagnostic performance of FDG-PET/CT separately in these two populations.

A limitation of the study is the lack of CT angiography (CTA). It is increasingly recognized that combined PET/CTA provides additional diagnostic information in various cardiovascular conditions such as infective endocarditis, vasculitis, and tube valve infection. ${ }^{3,20}$ Anatomical findings have great relevance in the diagnosis of vascular infection, as evidenced by their prominent role as a major MAGIC criterion. Integration of anatomical and metabolic information has the potential to improve both sensitivity and specificity of the examination.

The work of Zhang et al is a welcome addition to the body of literature supporting the complementary role of FDG-PET/CT in the evaluation of patients with 


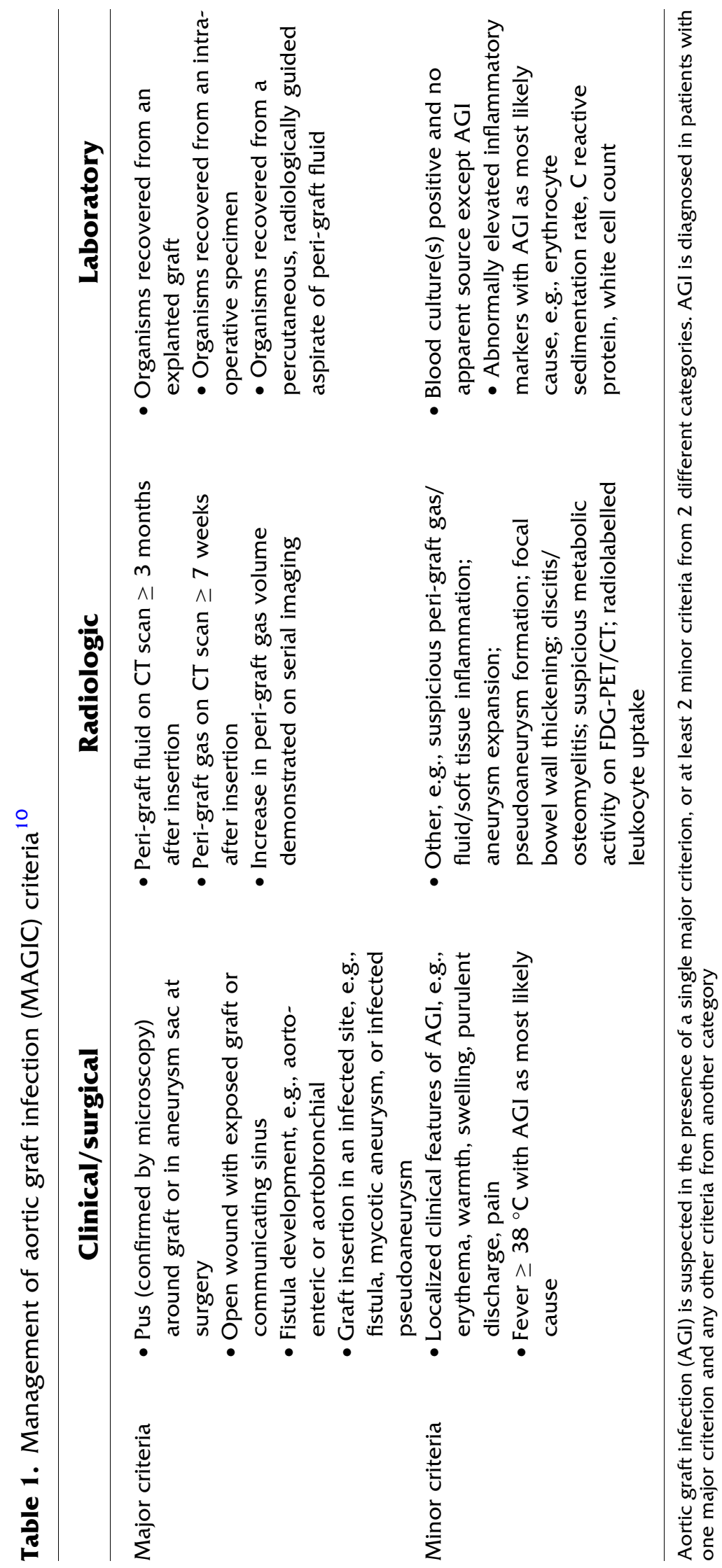


Table 2. Visual grade score as defined by Sah et al for the detection of vascular graft infection. ${ }^{18}$

\section{Visual grade}

\section{Definition}

\author{
Normal background activity \\ Mildly increased but diffuse uptake along the graft, less than twice the blood pool activity \\ Focal but mild uptake or strong diffuse uptake along the graft \\ Focal and intense uptake \\ Focal and intense uptake plus fluid collection/abscess formation
}

Grades 3-5 are considered positive for infection, while grades 1-2 are considered negative

suspected aortic graft infection. As we move forward, larger multicentric prospective trials evaluating not only the diagnostic accuracy but also the impact on management and outcomes are needed to further establish the role of FDG-PET/CT in the evaluation of patients with VGI. Ideally, such study would use combined PET/ CTA and focus on a specific type of procedure (open chest vs endovascular). As well, detailed analysis of cases with discordance between FDG-PET and the MAGIC criteria may provide a better understanding of the benefits and limitations of this modality. Finally, prospective studies designed specifically to clarify the role of FDG-PET in the evaluation of treatment response and establish the best follow-up methods are needed.

\section{Disclosures}

DJ has received consultant fees from AbbVie and Advanced Accelerator Applications. MP-G is supported by a Junior 1 Research Award from the Fonds de Recherche du Québec - Santé (FRQS).

\section{References}

1. Mc Ardle B, Shukla T, Nichol G, et al. Long-term follow-UP of outcomes with F-18-Fluorodeoxyglucose positron emission tomography imaging-assisted management of patients with severe left ventricular dysfunction secondary to coronary disease. Circ Cardiovasc Imaging 2016. https://doi.org/10.1161/CIRCIMA GING.115.004331.

2. Blankstein R, Osborne M, Naya M, et al. Cardiac positron emission tomography enhances prognostic assessments of patients with suspected cardiac sarcoidosis. J Am Coll Cardiol 2014;63:329-36. https://doi.org/10.1016/j.jacc.2013.09.022.

3. Pelletier-Galarneau M, Ruddy TD. PET/CT for diagnosis and management of large-vessel vasculitis. Curr Cardiol Rep 2019;21:34. https://doi.org/10.1007/s11886-019-1122-z.

4. Juneau D, Golfam M, Hazra S, et al. Positron emission tomography and single-photon emission computed tomography imaging in the diagnosis of cardiac implantable electronic device infection: A systematic review and meta-analysis. Circ Cardiovasc Imaging 2017. https://doi.org/10.1161/CIRCIMAGING.116.005772.

5. Lucinian YA, Lamarche Y, Demers P, et al. FDG-PET/CT for the detection of infection following aortic root replacement surgery. JACC Cardiovasc Imaging 2020;13:1447-9. https://doi.org/10.10 16/j.jcmg.2020.01.020.

6. Hobbs SD, Kumar S, Gilling-Smith GL. Epidemiology and diagnosis of endograft infection. J Cardiovasc Surg (Torino) 2010;51:5-14.

7. Chiesa R, Astore D, Frigerio S, et al. Vascular prosthetic graft infection: Epidemiology, bacteriology, pathogenesis and treatment. Acta Chir Belg 2002;102:238-47. https://doi.org/10.1080/ 00015458.2002.11679305.

8. O'Connor S, Andrew P, Batt M, Becquemin JP. A systematic review and meta-analysis of treatments for aortic graft infection. $\mathrm{J}$ Vasc Surg 2006;44:38-45. https://doi.org/10.1016/j.jvs.2006.02. 053 .

9. Baddour LM, Bettmann Michael A, Bolger Ann F, et al. Nonvalvular cardiovascular device-related infections. Circulation 2003;108:2015-31. https://doi.org/10.1161/01.CIR.0000093201.5 7771.47 .

10. Lyons OTA, Baguneid M, Barwick TD, et al. Diagnosis of aortic graft infection: A case definition by the management of aortic graft infection collaboration (MAGIC). Eur J Vasc Endovasc Surg 2016;52:758-63. https://doi.org/10.1016/j.ejvs.2016.09.007.

11. Chakfé N, Diener H, Lejay A, et al. Editor's choice - European Society for Vascular Surgery (ESVS) 2020 Clinical Practice Guidelines on the Management of Vascular Graft and Endograft Infections. Eur J Vasc Endovasc Surg 2020;59:339-84. https://doi. org/10.1016/j.ejvs.2019.10.016.

12. Kim S-J, Lee S-W, Jeong SY, et al. A systematic review and metaanalysis of 18F-fluorodeoxyglucose positron emission tomography or positron emission tomography/computed tomography for detection of infected prosthetic vascular grafts. J Vasc Surg 2019;70:307-13. https://doi.org/10.1016/j.jvs.2019.01.051.

13. Rojoa D, Kontopodis N, Antoniou SA, et al. 18F-FDG PET in the diagnosis of vascular prosthetic graft infection: A diagnostic test accuracy meta-analysis. Eur J Vasc Endovasc Surg 2019;57:292301. https://doi.org/10.1016/j.ejvs.2018.08.040.

14. Folmer EIR, Meijenfeldt GCIV, der Laan MJV, et al. Diagnostic imaging in vascular graft infection: A systematic review and metaanalysis. Eur J Vasc Endovasc Surg 2018;56:719-29. https://doi. org/10.1016/j.ejvs.2018.07.010.

15. Einspieler I, Mergen V, Wendorff H, et al. Diagnostic performance of quantitative and qualitative parameters for the diagnosis of aortic graft infection using [18F]-FDG PET/CT. J Nucl Cardiol 2020. https://doi.org/10.1007/s12350-019-02011-4. 
16. FitzGerald SF, Kelly C, Humphreys H. Diagnosis and treatment of prosthetic aortic graft infections: confusion and inconsistency in the absence of evidence or consensus. J Antimicrob Chemother 2005;56:996-9. https://doi.org/10.1093/jac/dki382.

17. Gordon BA, Flanagan FL, Dehdashti F. Whole-body positron emission tomography: Normal variations, pitfalls, and technical considerations. AJR Am J Roentgenol. 1997;169:1675-80. http s://doi.org/10.2214/ajr.169.6.9393189.

18. Sah B-R, Husmann L, Mayer D, et al. Diagnostic performance of 18F-FDG-PET/CT in vascular graft infections. Eur J Vasc Endovasc Surg 2015;49:455-64. https://doi.org/10.1016/j.ejvs.2014.12. 024 .
19. Treglia G, Slart RHJA, Glaudemans AWJM. Diagnostic performance and image interpretation of 18F-FDG PET/CT in aortic graft infection: Two sides of the same coin. J Nucl Cardiol 2020. h ttps://doi.org/10.1007/s12350-020-02029-z.

20. Pizzi MN, Roque A, Cuéllar-Calabria H, et al. 18F-FDG-PET/ CTA of prosthetic cardiac valves and valve-tube grafts: Infective versus inflammatory patterns. JACC Cardiovase Imaging 2016;9:1224-7. https://doi.org/10.1016/j.jcmg.2016.05.013.

Publisher's Note Springer Nature remains neutral with regard to jurisdictional claims in published maps and institutional affiliations. 